\title{
Anti-allergic activity of 2,4,6-trihydroxy-3-geranylacetophenone (tHGA) via attenuation of IgE-mediated mast cell activation and inhibition of passive systemic anaphylaxis
}

\begin{abstract}
tHGA, a geranyl acetophenone compound originally isolated from a local shrub called Melicope ptelefolia, has been previously reported to prevent ovalbumin-induced allergic airway inflammation in a murine model of allergic asthma by targeting cysteinyl leukotriene synthesis. Mast cells are immune effector cells involved in the pathogenesis of allergic diseases including asthma by releasing cysteinyl leukotrienes. The anti-asthmatic properties of tHGA could be attributed to its inhibitory effect on mast cell degranulation. As mast cell degranulation is an important event in allergic responses, this study aimed to investigate the anti-allergic effects of tHGA in cellular and animal models of IgE-mediated mast cell degranulation. For in vitro model of IgE-mediated mast cell degranulation, DNP-IgEsensitized RBL-2H3 cells were pre-treated with tHGA before challenged with DNP-BSA to induce degranulation. For IgE-mediated passive systemic anaphylaxis, Sprague Dawley rats were sensitized by intraperitoneal injection of DNP-IgE before challenged with DNP-BSA. Both in vitro and in vivo models showed that tHGA significantly inhibited the release of preformed mediators ( $\mathrm{b}$-hexosaminidase and histamine) as well as de novo mediators (interleukin-4, tumour necrosis factor-Ŭ prostaglandin D2 and leukotriene C4). Pre-treatment of tHGA also prevented IgE-challenged RBL-2H3 cells and peritoneal mast cells from undergoing morphological changes associated with mast cell degranulation. These findings indicate that tHGA possesses potent anti-allergic activity via attenuation of IgE-mediated mast cell degranulation and inhibition of IgE-mediated passive systemic anaphylaxis. Thus, tHGA may have the potential to be developed as a mast cell stabilizer for the treatment of allergic diseases in the future.
\end{abstract}

Keyword: tHGA; RBL-2H3; Anaphylaxis; Mast cell degranulation; IgE-mediated 